\title{
Vereinbarkeit von Familie und Beruf bei Beschäftigten im Gesundheitswesen als Handlungsfeld der Versorgungsforschung
} Compatibility of Work and Family Life of Employees in the Healthcare Sector: An Issue in Health Services Research

\section{() $\circledast \Theta$}

\section{Autoren}

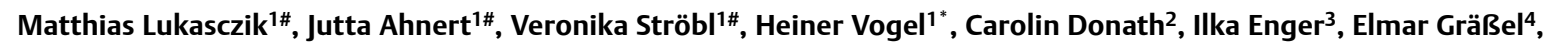
Lena Heyelmann ${ }^{5}$, Heidemarie Lux ${ }^{6}$, Jochen Maurer ${ }^{7}$, Dominik Özbe², Stefanie Spieckenbaum ${ }^{8}$, Elzbieta Voigtländer ${ }^{9}$, Manfred Wildner ${ }^{10}$, Andreas Zapf ${ }^{10}$, Angela Zellner ${ }^{10}$, Alfons Hollederer ${ }^{{ }^{*}}$

\section{Institute}

1 Abteilung für Medizinische Psychologie, Institut für Klinische Epidemiologie und Biometrie, JuliusMaximilians-Universität Würzburg, Würzburg

2 Zentrum für Medizinische Versorgungsforschung der Psychiatrischen Universitätsklinik Erlangen, FriedrichAlexander-Universität Erlangen-Nürnberg, Erlangen

3 Kassenärztliche Vereinigung Bayerns, München

4 Psychiatrische Universitätsklinik Erlangen, Zentrum für Medizinische Versorgungsforschung, Erlangen

5 Fachbereich Pflege, Katholische Stiftungshochschule München, München

6 Bayerische Landesärztekammer, München

7 Kassenärztliche Vereinigung Bayerns, SVS, München

8 Bayerisches Staatsministerium für Gesundheit und Pflege, München

9 Versorgungsqualität, Gesundheitsökonomie, Gesundheitssystemanalyse (GE6), Bayerisches Landesamt für Gesundheit und Lebensmittelsicherheit, Nürnberg

10 LGL, Bayerisches Landesamt für Gesundheit und Lebensmittelsicherheit, Oberschleißheim

\section{Schlüsselwörter}

Gesundheitssystem, Literaturübersicht, Versorgungsforschung, Vereinbarkeit von Familie und Beruf, Scoping Review

\section{Key words}

healthcare system, literature overview, health services research, work family compatibility, scoping review

\section{Bibliografie}

DOI http://dx.doi.org/10.1055/s-0043-101514

Online-Publikation: 18.5.2017

Gesundheitswesen 2018; 80: 511-521

(c) Georg Thieme Verlag KG Stuttgart · New York ISSN 0941-3790

\footnotetext{
\# gleichberechtigte Erstautoren

* gleichberechtigte Letztautoren
}

\author{
Korrespondenzadresse \\ Dr. Matthias Lukasczik \\ Institut für Klinische Epidemiologie und Biometrie \\ Abteilung für Medizinische Psychologie \\ Julius-Maximilians-Universität Würzburg \\ Klinikstraße 3 \\ 97070 Würzburg \\ matthias.lukasczik@uni-wuerzburg.de
}

\section{ZUSAMMENFASSUNG}

Hintergrund/Ziele Beschäftigte im Gesundheitswesen sind mit besonderen beruflichen Rahmenbedingungen und Anforderungen konfrontiert, die sich auf das Verhältnis von Familie und Arbeit/Beruf auswirken können und mittelbar auch die Qualität der Gesundheitsversorgung beeinflussen. Ziel des vorliegenden Beitrags ist es, einen Überblick über den Forschungsstand zu diesem Thema zu geben. Dieser ist für die Versorgungsforschung von Relevanz. Er kann als Ausgangspunkt dafür dienen, Strukturen im Gesundheitswesen (auch in ländlichen Regionen) dahingehend zu verändern, dass sie eine bessere Vereinbarkeit von Familie und Beruf ermöglichen.

Methode Es wurde eine systematische nationale und internationale Literaturrecherche in Form eines Scoping Review durchgeführt, um den aktuellen Forschungsstand zur Vereinbarkeit von Familie und Gesundheitsberuf zu dokumentieren. Als inhaltliche Suchkriterien wurden definiert: Vereinbarkeit von Beruf und Familie (allgemein); Vereinbarkeitserleben und -konflikte bei Gesundheitsberufen; Vereinbarkeit von Familie und Gesundheitsberuf im ländlichen Raum; Interventionsansätze zur Förderung der Vereinbarkeit von Beruf und Familie. Im Ergebnis eines mehrstufigen Selektionsprozesses wurden 145 Publikationen in die inhaltliche Auswertung einbezogen.

Ergebnisse Die dokumentierte Literatur bezieht sich schwerpunktmäßig auf die Berufsgruppen Ärzte und beruflich Pflegende, für andere Berufsgruppen liegen kaum Arbeiten vor. Die methodische Qualität der Studien ist meist niedrig, Metaanalysen liegen nicht vor. Mehrere Arbeiten dokumentieren eine Unzufriedenheit bei Ärzten und beruflich Pflegenden bzgl. 
Möglichkeiten der Vereinbarkeit von Familie und Beruf. Es konnten nur wenige Interventionsstudien zur Förderung der Vereinbarkeit von Familie und Beruf gefunden werden, diese sind wiederum nur teilweise spezifisch auf Gesundheitsberufe ausgerichtet. Defizite bestehen zudem hinsichtlich der Vernetzung mit Fragen der Verbesserung der Gesundheitsversorgung in ländlichen Gebieten.

Schlussfolgerung Zur Thematik „Vereinbarkeit von Familie und Gesundheitsberuf" existiert national wie international nur wenig systematische Forschung. Insbesondere zur Evaluation von Maßnahmen bestehen Defizite. Der Literaturüberblick ermöglicht die Ableitung von Ansatzpunkten, um eine Verbesserung der Vereinbarkeit von Familie und Beruf zu erreichen.

\footnotetext{
ABSTRACT

Background Healthcare professionals are confronted with specific work-related demands that influence work-family relations and might indirectly affect the quality of healthcare. This paper seeks to provide an overview of the current state of research on this topic of relevance to health services research. The overview may serve as a starting point for modifying structures in the healthcare system (especially in rural regions) with the aim of improving work-family compatibility.
}

Methods A systematic national and international literature search was conducted in terms of a scoping review. The following criteria/contents to be covered in publications were defined: work-family compatibility; work-family interface and work-family conflict in employees working in healthcare; healthcare professions in rural areas and links with work-family issues; interventions to improve work-family compatibility. 145 publications were included in the overview.

Results The available literature focuses on physicians and nursing staff while publications on other professions are largely lacking. The methodological quality of existing studies is mostly low, including a lack of meta-analyses. Several studies document dissatisfaction in physicians and nursing staff regarding reconciliation of work and family life. Only few intervention studies were found that seek to improve work-life compatibility; few of them focus on employees in healthcare. There are also deficits with respect to linking work-family issues with aspects of healthcare in rural areas.

Conclusions There is a shortage of systematic national and international research regarding work-family compatibility, especially when it comes to the evaluation of interventions. The overview provides starting points for improving work-family compatibility in healthcare.

\section{Einleitung}

Die Vereinbarkeit von Familie und Beruf ist ein Thema, das seit geraumer Zeit in Politik und Berufspraxis diskutiert wird, aber auch in der Forschung Aufmerksamkeit findet. In der internationalen Forschungsliteratur wird die Thematik unter Schlagwörtern wie „workfamily interface“ oder „work-family conflict“ (WFC) untersucht [1-4]. Letzterer beschreibt Interrollenkonflikte, die aus der Inkompatibilität der Anforderungen im beruflichen und im familiären Bereich erwachsen; WFC umfasst sowohl den Einfluss arbeits- bzw. berufsbezogener Anforderungen auf den familiären Lebensbereich (work interfering with family, WIF) als auch die Auswirkungen familienbezogener Anforderungen auf Beruf bzw. Arbeit (family interfering with work, FIW) [5-7]. Konsistent wurde belegt, dass sich Belastungen im Arbeits- und Berufsbereich stärker auf den Bereich Familie auszuwirken scheinen (WIF) als umgekehrt. Als ein wichtiger Moderator erwies sich das Vorhandensein von Kindern: je höher der Anteil an Eltern in den einzelnen Studien, umso höher war die positive Korrelation zwischen arbeitsbezogenem Stress und FIW sowie zwischen Stress und WIF [2,3].

Für den Kontext des Gesundheitswesens ist die Thematik der Vereinbarkeit von Familie und Beruf von Bedeutung, weil hier Fragen der Qualität der gesundheitlichen Versorgung auf unterschiedlichen Ebenen berührt werden. Dies betrifft unter anderem die spezifischen Formen von Anforderungen und Belastungen der im Gesundheitswesen tätigen Professionen (im Folgenden unter dem Begriff „Gesundheitsberufe“ zusammengefasst) wie z. B. Schichtdienste, die individuelle Zufriedenheit und Leistungsfähigkeit von Ärzten, beruflich Pflegenden, Assistenzberufen oder therapeutischen Berufen (z. B. Physiotherapie, Ergotherapie, Psychotherapie) oder auch die Gewährleistung einer ausreichenden (medizinischen)
Versorgungsdichte in ländlichen Gebieten in Abhängigkeit von der Verfügbarkeit einer familienfreundlichen Infrastruktur.

Die Kenntnis des aktuellen Forschungsstands zur Vereinbarkeit von Familie und Gesundheitsberuf und der besonderen Rahmenbedingungen und Anforderungen für Gesundheitsberufe ist auch für die Versorgungsforschung von besonderer Relevanz. Hieraus können Ansatzpunkte für mögliche Modellprojekte oder Interventionen mit der Zielsetzung einer Förderung der Vereinbarkeit von Familie und Beruf abgeleitet werden, die in den Strukturen des Gesundheitssystems erprobt und evaluiert werden können.

Im vorliegenden Beitrag wird eine Übersicht zur Thematik der Vereinbarkeit von Familie und Beruf bei Angehörigen von Gesundheitsberufen gegeben. Grundlage dieser Übersicht ist eine Expertise, die durch das Bayerische Staatsministerium für Gesundheit und Pflege (StMGP) im Rahmen der Landesarbeitsgemeinschaft Gesundheitsversorgungsforschung (LAGeV) in Auftrag gegeben und von dieser begleitet wurde [8]. Die Expertise hatte zum Ziel, den Ist-Stand bzgl. des Themas „Vereinbarkeit von Familie und Gesundheitsberuf" zu analysieren und hieraus Zielsetzungen und Ansatzpunkte für Modellprojekte zur Optimierung der regionalen Versorgungsstrukturen abzuleiten.

Der vorliegende Beitrag stellt einen Teil der Ergebnisse dieser Expertise vor. Im Beitrag wurde der Schwerpunkt auf die zusammenfassende Darstellung des Wissens- und Forschungsstands gelegt.

\section{Methodik}

Angesichts der Komplexität des Themenfelds und des weitgehenden Fehlens von systematischen Übersichtsarbeiten wurde die Lite- 
raturrecherche in Form eines Scoping Review durchgeführt [9-11]. Hierbei werden der Wissensstand, relevante Konzepte und Forschungsdefizite im interessierenden Themenbereich systematisch zusammengestellt und aufgezeigt, wobei auch Publikationen unterschiedlicher methodischer Güte einbezogen werden. Ziel ist es, einen möglichst vollständigen Überblick zu einer explorativen bzw. komplexen Thematik zu generieren, welcher als Vorarbeit für differenzierte Analysen (etwa systematische Reviews), aber auch als Informationsgrundlage für Entscheidungsträger und Akteure im Gesundheitssystem dienen kann.

Zwischen Juli und Oktober 2015 wurde eine systematische nationale und internationale Literaturrecherche durchgeführt. Hierbei wurden die wissenschaftlichen Datenbanken PUBMED, MEDLINE, PSYCINFO, PSYNDEX und Cochrane Database of Systematic Reviews einbezogen, ferner Google Scholar. Zudem wurden die Literaturangaben bereits identifizierter Publikationen gesichtet, um dort weitere für die Thematik relevante Veröffentlichungen aufzufinden (Citation Tracking). Darüber hinaus wurden bei fehlenden Veröffentlichungen von Studienergebnissen verschiedene medizinische Fachgesellschaften und Berufsverbände direkt kontaktiert. Des Weiteren erfolgte eine Recherche in der Suchmaschine Google zur Identifizierung nicht primär wissenschaftlicher Quellen wie Broschüren oder Ratgeber zur Thematik, soweit vorhanden, auch mit regionalem oder lokalem Bezug.

Es wurden zunächst die folgenden Themenbereiche im Sinne inhaltlicher Kriterien definiert:

- Vereinbarkeit von Beruf und Familie (allgemein)

- Vereinbarkeitserleben und -konflikte bei Gesundheitsberufen (international, Deutschland)

- Vereinbarkeit von Familie und Gesundheitsberuf im ländlichen Raum (international, Deutschland)

- Interventionsansätze zur Förderung der Vereinbarkeit von

Beruf und Familie (international, Deutschland)

Im Rahmen der Literaturrecherche wurden die in > Tab. 1 aufgeführten Suchbegriffe und Schlagwörter verwendet (auch Trunkierungen und Kombinationen von Suchbegriffen (AND-Verknüpfung)).

Die anhand der Suchbegriffe identifizierten Veröffentlichungen wurden zunächst anhand von Titel und Abstract geprüft, inwieweit sie einem (oder mehreren) der oben genannten Themenbereiche zugeordnet werden können. Die so ausgewählten Publikationen wurden dann vollständig durchgesehen (Volltext) und wiederum auf Relevanz hinsichtlich der inhaltlichen Kriterien geprüft. Im Ergebnis dieses Selektions-/Extraktionsprozesses wurden insgesamt 145 Publikationen in die weiteren Auswertungen und Ergebnisdarstellungen einbezogen.

Gemäß dem Ziel, den aktuellen Kenntnisstand inhaltlich darzustellen und im Sinne eines Scoping Review zusammenfassend zu referieren (s. o.), wurden dementsprechend nicht nur empirische Originalarbeiten, sondern auch Übersichtsarbeiten und zusammenfassende Darstellungen berücksichtigt.

\section{Ergebnisse}

Um eine komprimierte Darstellung der Thematik zu ermöglichen, wird in dieser Arbeit nicht auf alle Publikationen bzw. Publikationstypen eingegangen, die im Rahmen der Expertisenerstellung gefun-
- Tab. 1 Suchbegriffe im Rahmen der Literaturrecherche.

\begin{tabular}{|l|l|}
\hline Englische Suchbegriffe & Deutsche Suchbegriffe \\
\hline - work-family & - Beruf, Familie \\
- conflict & - Heilberufe, Gesundheitsberufe \\
- roles & - Gesundheitswesen \\
- facilitation & - Vereinbarkeit; vereinbar \\
- enhancement & - Arzt; ärztlich \\
- satisfaction & - Medizin; medizinisch \\
- enrichment & - Medizinische Fachgesellschaften \\
- stress & - Therapeut; therapeutisch \\
- health/healthcare & - Physiotherapeut/Ergotherapeut \\
- physician & - Psychotherapeut/Psychiater \\
- intervention & - Zahnarzt \\
- promotion & - Pflege(kraft), Pflegeeinrichtun- \\
- program & gen \\
- balance/imbalance & - Krankenhaus, Klinik \\
- double duty & - Medizinische Fachangestellte \\
- caregiver/caregiving & - Rettungsdienst/Sanitäter, \\
- nurse & Notdienst \\
- pharmacist & - Hebamme \\
- (occupational, physio) & - Apotheker \\
therapist, physical therapist & - Programm \\
- paramedic & - Intervention \\
- emergency medical & - Pflege Angehöriger \\
- assistant/technician & - Vereinbarkeit Beruf und Pflege \\
- midwife & Angehöriger \\
- emergency medical & - Befragung, Umfrage \\
- rural/urban & - ländlich/ländlicher Raum/ \\
- remote & strukturschwach \\
- medically underserved & \\
- physician shortage (area) & \\
- recruitment, retention & \\
\hline
\end{tabular}

den wurden. Unberücksichtigt bleiben nicht primärwissenschaftliche Quellen wie Broschüren und Informationsmaterialien zur Thematik oder Fördermaßnahmen von Institutionen und Körperschaften (Fachgesellschaften, Kassenärztliche Vereinigungen). Ferner werden die im Rahmen der Expertise identifizierten Studien und Befragungen mit Studierenden der Medizin hier nicht dargestellt.

\section{Vereinbarkeit von Familie und Beruf im Kontext von Gesundheitsberufen: Internationale Befunde}

Für Gesundheitsberufe wurden keine spezifischen systematischen Übersichtsarbeiten zum Thema identifiziert. Es konnten wenige internationale Studien bei Ärzten bzw. beruflich Pflegenden gefunden werden. Zu anderen Gesundheitsberufen (z. B. Physiotherapeuten, Ergotherapeuten, Hebammen) liegen nur vereinzelt Studien vor.

Studien aus Australien, Frankreich, Malaysia, den Niederlanden und Ungarn zeigen, dass bei Ärzten verschiedener Fachrichtungen das Erleben von Vereinbarkeitskonflikten positiv mit Burnout assoziiert ist, aber auch mit anderen ungünstigen Outcomes wie Kündigungsbereitschaft oder Beeinträchtigungen der subjektiven Gesundheit korreliert [12-16]. Für beruflich Pflegende ist in internationalen Studien belegt, dass Vereinbarkeitskonflikte mit stärkerer negativer Gestimmtheit, emotionaler Erschöpfung, der Absicht zu kündigen bzw. den Arbeitsplatz zu wechseln, aber auch mit verschlechterten gesundheitlichen Parametern (Schmerzsymptomatik, erhöhter kardiometabolischer Risikoscore) einhergehen [17-21].

Die Ergebnisse einer belgischen Studie zu organisatorischen Faktoren in Krankenhäusern, die sich auf das Erleben der Arbeitstätig- 
keit bei beruflich Pflegenden auswirken, implizieren, dass als attraktiv erlebte Kliniken viele Merkmale eines „healthy workplace“ [22] aufweisen (u. a. geringeres Maß an Arbeitsbelastung/-überlastung und Rollenkonflikten, bessere erlebte Vereinbarkeit von Beruf und Familie, mehr Entscheidungsspielräume) [23]. Die Vereinbarkeit von Familie und Gesundheitsberuf wird wesentlich von den Elementen eines solchen Arbeitsplatzes beeinflusst.

In der umfassenden europaweiten NEXT-Studie (Nurses Early Exit Study) mit knapp 40000 befragten beruflich Pflegenden (auch aus Deutschland) wurden Arbeitsbedingungen und Gründe für das Aufgeben des Pflegeberufs untersucht. Im Speziellen wurden auch Konflikte zwischen Arbeits- und Familienleben analysiert [21]. Deren Ausprägung lag im Vergleich mit den anderen berücksichtigten Ländern in Deutschland im mittleren Bereich und sehr nahe am Gesamtmittelwert. Ähnlich wie in den oben genannten Studien waren negative Einflüsse des Lebensbereichs „Arbeit“ auf die Familie stärker ausgeprägt als umgekehrt. Beruflich Pflegende mit Versorgungsverantwortung für Kinder gaben deutlichere ArbeitFamilie-Konflikte an. Wesentliche Geschlechterunterschiede fanden sich nicht.

Die Gruppe der Mitarbeiter in Gesundheitsberufen, die sowohl Kinder als auch (ältere) pflegebedürftige Angehörige versorgen (in der Literatur auch als „double/triple duty caregiving“ bezeichnet $[24,25])$, hat in der Forschung bislang wenig Aufmerksamkeit gefunden. Erst in jüngster Zeit haben sich Studien aus Kanada, den USA und den Niederlanden mit beruflich Pflegenden in Mehrfachverantwortung beschäftigt. Diese kann - im Vergleich zu beruflich Pflegenden ohne Verantwortung für Kinder oder pflegebedürftige Angehörige - mit verschiedenen Beeinträchtigungen des Befindens und der subjektiven Gesundheit einhergehen [24, 26, 27].

Für nichtärztliche therapeutische Berufe bzw. Assistenzberufe lagen kaum Studien vor, die sich mit der Thematik der Vereinbarkeit von Familie und Beruf beschäftigt haben. Es fehlen systematische und repräsentative Erhebungen.

\section{Vereinbarkeit von Familie und Beruf im Kontext von Gesundheitsberufen: Befunde aus Deutschland}

Es wurden kaum Studien aus Deutschland gefunden, die gezielt das Thema Vereinbarkeitskonflikte bei Gesundheitsberufen analysieren. In einer Studie wurde die Häufigkeit von Vereinbarkeitskonflikten bei 269 deutschen Krankenhausärzten untersucht [28]. Es wurde eine signifikant höhere Ausprägung als in der deutschen Allgemeinbevölkerung festgestellt. Das Konflikterleben war mit Burnout, Stresserleben und Kündigungsabsichten korreliert. Befragungen von beruflich Pflegenden zeigen, dass eine mangelnde erlebte Vereinbarkeit von Beruf und Familie mit Faktoren wie beruflicher bzw. Arbeitsplatzunzufriedenheit [29, 30] oder einem schlechteren Gesundheitszustand [31] korreliert ist.

Verschiedentlich untersucht wurden auch Fragen der Karrieregestaltung bzw. Karriereverläufe. In einer Studie zu den Auswirkungen der Elternzeit bei 406 Beschäftigten an einer medizinischen Hochschule auf den weiteren Karriereverlauf wurde belegt, dass 51 \% der Beschäftigten nach Rückkehr aus der Elternzeit eine signifikante Änderung ihrer Arbeitsaufgaben erlebten, 17 \% der Führungskräfte ihren Status verloren und $58 \%$ einen Arbeitgeberwechsel erwogen [32]. Im Report Versorgungsforschung gaben 76\% der befragten Ärztinnen und $18 \%$ der Ärzte an, dass sich ihre Weiterbildung durch die Kinderbetreuung verzögert habe [33]. 74\% der Medizinerinnen und $9 \%$ der Mediziner unterbrachen für die Betreuung der eigenen Kinder ihre Weiterbildung, 15\% der Ärztinnen und $1 \%$ der Ärzte beendeten diese hierfür sogar gänzlich. In mehreren Befragungen von Ärzten zeigte sich, dass ein Großteil der Befragten eine bessere Vereinbarkeit von Beruf und Familie präferiert und Arbeitszeiten gerne reduzieren würde [34-36]. Etwa die Hälfte der Ärzte äußerte sich unzufrieden mit den aktuell existierenden Möglichkeiten der Kinderbetreuung [37, 38] und wünschte sich eine bessere Abstimmung der Kinderbetreuungsmöglichkeiten mit ihren Arbeitszeiten. Ähnliche Ergebnisse finden sich auch bei der Befragung von beruflich Pflegenden [30]. > Tab. 2 enthält einen Überblick über Befragungen von Ärzten und Zahnärzten zur Vereinbarkeit von Familie und Beruf in Deutschland.

Es existieren nur wenige Erhebungen, die den aktuellen IstStand hinsichtlich Kinderbetreuungs- und Teilzeitarbeitsmöglichkeiten an deutschen Kliniken bzw. Pflegeeinrichtungen erfassen. In einer Befragung des Deutschen Ärztinnenbunds zur Kinderbetreuung an deutschen Kliniken und Krankenhäusern [51] gaben $15 \%$ der 721 befragten Kliniken an, dass sie in ihrem Haus eine Kinderbetreuung anbieten. Nach Zahlen des Deutschen Krankenhausinstitutes (DKI) boten im Jahr 2010 lediglich 19\% der deutschen Krankenhäuser betriebliche Belegplätze in Kinderbetreuungseinrichtungen der Umgebung an. 15 \% verfügten über eigene Kinderbetreuungseinrichtungen [52]. Dabei bewerteten 49\% der befragten Krankenhäuser die Platzzahlen der Kinderbetreuungseinrichtungen als bedarfsgerecht. Für $31 \%$ der Kliniken deckten diese teilweise, für $18 \%$ hingegen eher nicht den Bedarf.

Das DKI untersuchte zudem 2008 die Familienorientierung im stationären Sektor mit dem Ergebnis, dass $40 \%$ der Kliniken der Vereinbarkeit von Familie und Arztberuf einen mittleren bis hohen Stellenwert in ihrer Unternehmensstrategie zuwiesen [53]. Für 12\% der Krankenhäuser spielte dies keine Rolle. Der Stellenwert der Vereinbarkeit von Familie und Beruf in der Unternehmensstrategie hing dabei signifikant von der Größe der Krankenhäuser ab: während zwei Drittel der Krankenhäuser ab 600 Betten dieser einen eher hohen Stellenwert zurechneten, war dies bei Kliniken bis 300 Betten nur bei einem Drittel der Fall.

Die Industrie- und Handelskammer Berlin und die Universität Kiel untersuchten in Zusammenarbeit mit dem Bundesverband privater Anbieter sozialer Dienste die Vereinbarkeit von Beruf und Familie in 350 ambulanten und stationären Pflegeeinrichtungen [54]. In $78 \%$ der Einrichtungen war die Betreuung von Kindern und Jugendlichen wichtig für die Arbeitszufriedenheit. Fast die Hälfte der Einrichtungen sah auch in einer Gewährleistung der Pflege von Angehörigen einen wichtigen Faktor für die Arbeitszufriedenheit. $62 \%$ der Einrichtungen boten spezielle Arbeitszeitmodelle für Beschäftigte mit Familienpflichten an. Etwa ein Drittel der Einrichtungen unterstützte die Mitarbeiter aktiv bei der Kinderbetreuung. 70\% der Einrichtungen gaben Hilfen bei Wiedereinstieg in den Beruf, Servicedienste für Familien waren dagegen nur bei $23 \%$ in den Routinebetrieb integriert. 75 \% der Einrichtungen wünschten sich Kooperationen mit externen Dienstleistern zur Kinderbetreuung. 
- Tab. 2 Befragungen von Ärzten und Zahnärzten zur Vereinbarkeit von Familie und Beruf in Deutschland.

\begin{tabular}{|c|c|c|}
\hline Quelle & Stichprobe & Themenfelder \\
\hline \multicolumn{3}{|l|}{ Ärzte } \\
\hline Gensch (2010) [33] & $\begin{array}{l}\mathrm{n}=1010 \text { Ärzte in Bayern verschiedener } \\
\text { Fachrichtungen (Klinik und Niederlassung) } \\
\text { (Befragungszeitraum: 2002-2004) }\end{array}$ & $\begin{array}{l}\text { - Vereinbarkeit von Beruf und Familie } \\
\text { - zukünftiges Tätigkeitsfeld } \\
\text { - Niederlassungsgründe } \\
\text { - familienverträgliche Arbeitszeiten } \\
\text { - Zufriedenheit mit Kinderbetreuungsangeboten } \\
\text { - Weiterbildung } \\
\text { - Karriereunterbrechung }\end{array}$ \\
\hline Flaig (2014) [39] & $\begin{array}{l}\mathrm{n}=121 \text { Ärzte verschiedener Fachrichtungen } \\
\text { der medizinischen Fakultät der Universität } \\
\text { Ulm }\end{array}$ & $\begin{array}{l}\text { - Vereinbarkeit von Familie und Beruf } \\
\text { - Arbeitsanforderungen, Belastungen } \\
\text { - Arbeitsunzufriedenheit } \\
\text { - Konflikterleben zwischen Arbeits- und Familienleben } \\
\text { - Angststörungen oder Depressionen } \\
\text { - Teilzeitmodelle }\end{array}$ \\
\hline $\begin{array}{l}\text { Bühren (2010), Bühren \& } \\
\text { Buchalik (2008), Bühren \& } \\
\text { Tschörtner (2011) [34, 40, 41] }\end{array}$ & $\begin{array}{l}n=1200 \text { Ärztinnen (Klinik und Niederlas- } \\
\text { sung) }\end{array}$ & $\begin{array}{l}\text { - Vereinbarkeit von Beruf und Familie } \\
\text { - geschlechterspezifische und gesellschaftliche Hemmnisse und } \\
\text { Schwierigkeiten } \\
\text { - Hindernisse im Beruf } \\
\text { - Zufriedenheit im Beruf } \\
\text { - Vergleich niedergelassene Ärztinnen vs. Klinik } \\
\text { - Vergleich Ärztinnen mit und ohne Kinder } \\
\text { - flexible Arbeitseinteilung } \\
\text { - Ärztinnen mit Kind } \\
\text { - Teilzeitmodelle }\end{array}$ \\
\hline Fischer (2014) [42] & $\begin{array}{l}\mathrm{n}=627 \text { Interviews mit niedergelassenen } \\
\text { und angestellten ambulant tätigen } \\
\text { Ärztinnen und Ärzten, Psychologischen } \\
\text { Psychotherapeuten sowie Kinder- und } \\
\text { Jugendlichenpsychotherapeuten }\end{array}$ & $\begin{array}{l}\text { - Vergleich Hausarzt, Facharzt, Psychologischer Psychotherapeut } \\
\text { - Vereinbarkeit Beruf und Familie } \\
\text { - Arbeitszeitbelastung/Woche } \\
\text { - Reduktion der Arbeitszeit } \\
\text { - Vorteile der niedergelassenen Tätigkeit von Gemeinschaftspraxen und } \\
\text { Praxisgemeinschaften }\end{array}$ \\
\hline $\begin{array}{l}\text { Deutsche Gesellschaft für } \\
\text { Kardiologie (2014) [43] }\end{array}$ & Kardiologen ( $\mathrm{N}$ nicht angegeben) & - Vereinbarkeit von Familie und Beruf \\
\hline Hancke et al. (2012) [44] & $\begin{array}{l}\mathrm{n}=1037 \text { Mitglieder der Deutschen } \\
\text { Gesellschaft für Gynäkologie und } \\
\text { Geburtshilfe: } 75 \% \text { Frauenärztinnen und } \\
25 \% \text { Frauenärzte }\end{array}$ & $\begin{array}{l}\text { - Vereinbarkeit von Familie und Beruf/beruflicher Karriere } \\
\text { - außerfamiliäre Kinderbetreuungsmöglichkeiten }\end{array}$ \\
\hline $\begin{array}{l}\text { Hartmannbund (2014), Flintrop } \\
(2015)[35,45,46]\end{array}$ & $\begin{array}{l}\mathrm{n}=1400 \text { Assistenzärzte (Klinik und } \\
\text { angestellt ambulant tätig) }\end{array}$ & $\begin{array}{l}\text { - Vereinbarkeit von Familie und Beruf } \\
\text { - strukturierte Schichtdienstmodelle statt Bereitschaftsdienste } \\
\text { - zukünftiges Tätigkeitsfeld (selbstständig vs. angestellt) } \\
\text { - Opt-Out-Regelung } \\
\text { - Teilzeitanstellung } \\
\text { - Mutterschutz, Elternzeit } \\
\text { - beruflicher Wiedereinstieg } \\
\text { - finanzieller Nachteilsausgleich } \\
\text { - Weiterbildung } \\
\text { - Nachteile aufgrund von Schwangerschaft und Familiengründung }\end{array}$ \\
\hline Engelmann (2015) [32] & $\begin{array}{l}\mathrm{n}=406 \text { (91 Ärzte, } 18 \text { andere Akademiker, } \\
284 \text { Nicht-Akademiker) } \mathrm{n}=63 \text { Abteilungs- } \\
\text { leiter der Medizinischen Hochschule } \\
\text { Hannover (MHH); plus Interviews mit } 3 \\
\text { Personalchefs }\end{array}$ & $\begin{array}{l}\text { Elternzeit bzw. Rückkehr aus der Elternzeit und Folgen für die berufliche } \\
\text { Situation bzgl. } \\
\text { - Änderung der Arbeitsaufgaben } \\
\text { - Verlust an Führungsaufgaben, Statusverlust } \\
\text { - erhöhter Fluktuation, Arbeitsplatzwechsel } \\
\text { - Zunahme an Arbeitsbelastung } \\
\text { - Karriereperspektiven } \\
\text { - unterschiedlicher Wahrnehmung/Bewertung der Veränderungen im } \\
\text { Tätigkeitsbereich durch Beschäftigte und Vorgesetzte }\end{array}$ \\
\hline Oberlander (2010) [47] & $\begin{array}{l}\mathrm{n}=1308 \text { in Kliniken tätige und niedergelas- } \\
\text { sene Ärzte, seit höchstens } 10 \text { Jahren } \\
\text { approbiert }\end{array}$ & $\begin{array}{l}\text { - Work-Life-Balance } \\
\text { - Vergleich nicht-kurativ tätige Ärzte, niedergelassene Ärzte, Ärzte in } \\
\text { Kliniken und ambulant angestellte Ärzte }\end{array}$ \\
\hline Bestmann et al. (2010) [37] & $\begin{array}{l}\mathrm{n}=2165 \text { Ärzte verschiedener Fachrichtun- } \\
\text { gen (Klinik und Niederlassung) }\end{array}$ & $\begin{array}{l}\text { - wöchentliche Arbeitszeit } \\
\text { - Zufriedenheit mit der Kinderbetreuung } \\
\text { - Vergleich Kliniken und ambulanter Bereich }\end{array}$ \\
\hline
\end{tabular}


Tab. 2 Fortsetzung

\begin{tabular}{|c|c|c|}
\hline Quelle & Stichprobe & Themenfelder \\
\hline Barth et al. (2015) [48] & $\begin{array}{l}\text { Ärzte in der Weiterbildung (ÄiW; Klinik und } \\
\text { angestellt in Niederlassung); Befragung zu } \\
\text { Zukunftsperspektiven: } 3 \text { Fokusgruppen } \\
\text { ( } n=13 \text { ), Telefoninterviews ( } n=24) \text {, } \\
\text { Onlinebefragung ( } n=132)\end{array}$ & $\begin{array}{l}\text { - Teilzeitmodelle } \\
\text { - Praxisgemeinschaft } \\
\text { - Niederlassungsmotive }\end{array}$ \\
\hline \multicolumn{3}{|l|}{ Zahnärzte } \\
\hline $\begin{array}{l}\text { Bayerische Landesärztekammer } \\
\text { (2015) [49] }\end{array}$ & $\mathrm{n}=1300$ Zahnärztinnen in Bayern & $\begin{array}{l}\text { - Vereinbarkeit Beruf und Familie } \\
\text { - Vergleich niedergelassene, angestellte und verbeamtete Zahnärztinnen } \\
\text { - Doppelbelastung } \\
\text { - Wiedereinstieg } \\
\text { - Zufriedenheit mit Einrichtungen zur Kinderbetreuung } \\
\text { - Teilzeitmodelle }\end{array}$ \\
\hline $\begin{array}{l}\text { Institut für freie Berufe } \\
\text { Nürnberg, Bayerische } \\
\text { Landeszahnärztekammer } \\
\text { (2011) [50] }\end{array}$ & Zahnärztinnen (n nicht angegeben) & $\begin{array}{l}\text { - Vereinbarkeit Beruf und Familie } \\
\text { - Vergleich niedergelassene vs. angestellte Zahnärztinnen } \\
\text { - Gründe für Schwierigkeiten bei der Vereinbarkeit von Beruf und } \\
\text { Familie (Betreuungsmöglichkeiten für Kinder, finanzielle Unterstüt- } \\
\text { zung, Organisation von Vertretungen, Niederlassungsrisiko) }\end{array}$ \\
\hline Heyer (2014) [38] & n=200 Zahnärzte und Praxispersonal & $\begin{array}{l}\text { - Vereinbarkeit von Arbeitszeiten und Kinderbetreuung } \\
\text { - Nutzungswünsche für Kinderbetreuungsangebote }\end{array}$ \\
\hline
\end{tabular}

\section{Vereinbarkeit von Familie und Gesundheitsberuf und Tätigkeit im ländlichen Raum}

In den im Rahmen der Literaturrecherche selektierten Studien zur Tätigkeit in Gesundheitsberufen im ländlichen Raum wurde die Frage der Vereinbarkeit von Familie und (Gesundheits-)Beruf nicht behandelt. Eine internationale Literaturübersicht zu Ansätzen zur Gesundheitsversorgung in ländlichen Gebieten thematisierte die Vereinbarkeit von Familie und Beruf nicht explizit, wenngleich einige der Ansätze Entlastungen hinsichtlich der Arbeitszeit implizieren, sodass hieraus indirekt ein Benefit für die Work-Life-Balance entstehen kann (z. B. Delegieren/Neustrukturierung von Aufgaben, Verbesserung der Schnittstellen, Telemedizin) [55]. Auch in internationalen Studien, die Modellprojekte und deren Evaluation zu Fragen der Tätigkeit im ländlichen Raum vorstellen [56-59], fehlt der Bezug zur Vereinbarkeit von Familie und Gesundheitsberuf.

Studien wurden vorrangig in Ländern wie z. B. Australien und Kanada durchgeführt. In Bezug auf niederlassungsfördernde und -hemmende Faktoren für Ärzte in Deutschland fassen Langer und Kollegen [60] die Ergebnisse von 10 Studien in einer Literaturübersicht zusammen. Unter die identifizierten nicht-monetären Faktoren, die für eine Niederlassung bedeutsam sind, fallen Rahmenbedingungen für die Familie (z. B. berufliche Möglichkeiten für den Lebenspartner, Betreuungs- und Schulangebote für Kinder), berufliche Kooperationsmöglichkeiten (z. B. fachlicher Austausch mit Kollegen, gemeinsame Nutzung von Geräten) sowie berufliche Verpflichtungen (z. B. Zahl der Bereitschaftsdienste) und Arbeitsbedingungen (z. B. Zeit pro Patient). In zwei der Studien wurden Geschlechtsunterschiede untersucht; demzufolge spielen für Männer die Arbeitsbedingungen eine größere, die Vereinbarkeit von Familie und Beruf hingegen eine geringere Rolle als für Frauen.

Ansätze im deutschen Gesundheitssystem zur Delegation (haus-)ärztlicher Aufgaben an speziell qualifizierte Fachkräfte, die u. a. zu einer Verbesserung der Versorgung im ländlichen Raum und einer Entlastung von Ärzten beitragen sollen, wurden nur teilweise evaluiert. Hierzu zählen etwa die Modellprojekte VerAH (Versor- gungsassistentin in der Hausarztpraxis) und AGnES (Arzt-entlastende, Gemeinde-nahe, E-Health-gestützte, Systemische Intervention) $[61,62]$. Fragen der Vereinbarkeit von Beruf und Familie wurden auch hier nicht thematisiert.

\section{Interventionsansätze zur Förderung der Vereinbarkeit von Familie und Beruf}

Es konnten nur wenige Interventionsstudien identifiziert werden, die die Förderung der Vereinbarkeit von Familie und Beruf zum Inhalt haben und die wiederum nur teilweise Professionen im Gesundheitswesen in den Mittelpunkt rücken ( $\triangleright$ Tab. 3). Die identifizierten Interventionen setzen an unterschiedlichen Ebenen an (individuelle Ebene; Teamebene; Organisationsebene). Reviews zu evidenzbasierten Interventionen wurden nicht gefunden. Es existiert lediglich ein Cochrane-Review zu den Effekten (zeitlich) flexibler Arbeitsbedingungen (u. a. flexible Arbeitszeiten, Teilzeittätigkeit, zeitlich befristete Verträge) auf Befindlichkeit und Gesundheit von Arbeitnehmern [63]. In diesem Review wird auf eine geringe Zahl geeigneter Studien, methodische Schwächen bestehender Arbeiten und Defizite in der Forschung in diesem Bereich hingewiesen. Es wurden nicht gezielt Gesundheitsberufe einbezogen und Vereinbarkeitskonflikte nicht analysiert. In der vorliegenden Primärliteratur wird ebenfalls das weitgehende Fehlen von entsprechenden Ansätzen konstatiert.

Existierende Untersuchungen zu den Assoziationen von Vereinbarkeitskonflikten und unterschiedlichen Formen der Arbeitsgestaltung weisen darauf hin, dass flexible Arbeitsbedingungen (in zeitlicher und räumlicher Hinsicht) begrenzte Potenziale zur Reduktion von Vereinbarkeitsproblemen haben [64, 65]. In einer deutschen Erhebung äußerten die Befragten überwiegend, dass flexible Arbeitszeiten als wichtig und hilfreich für eine bessere Vereinbarkeit von Familie und Beruf erlebt werden [66].

Die identifizierten Interventionsansätze bzw. -studien sind in - Tab. 3 aufgeführt. 


\begin{tabular}{|c|c|c|c|c|c|}
\hline & 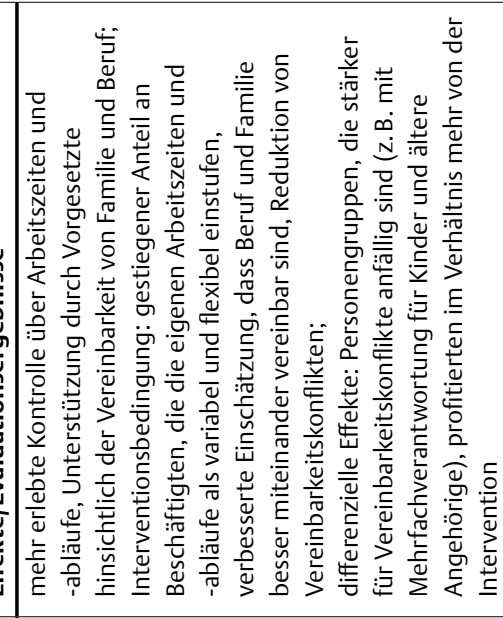 & 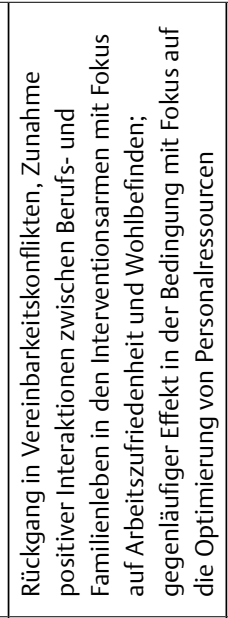 & 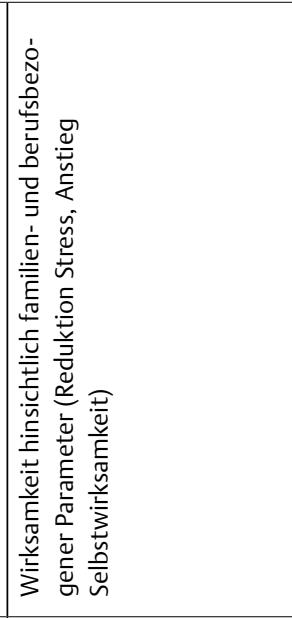 & 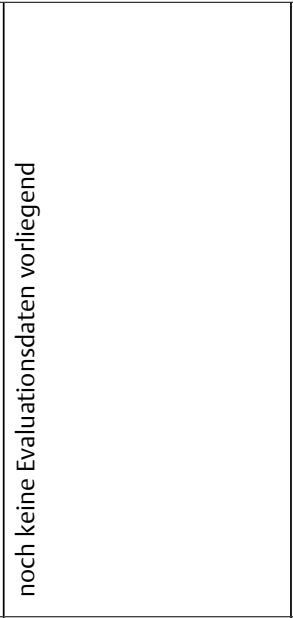 & 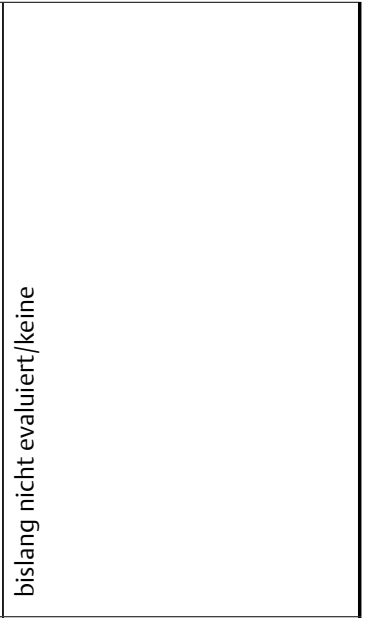 \\
\hline & 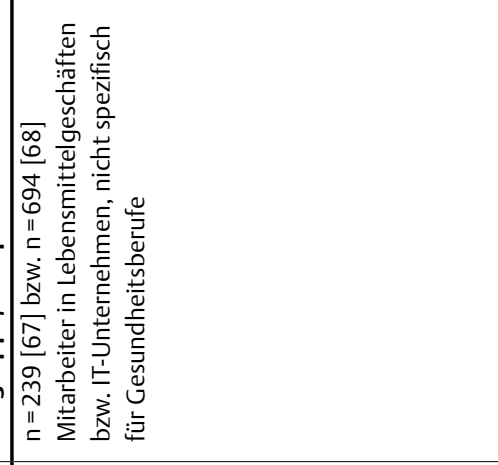 & 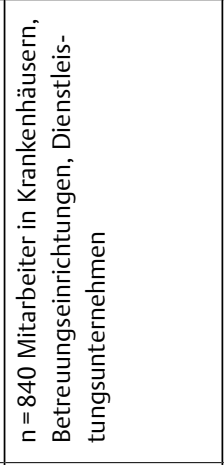 & 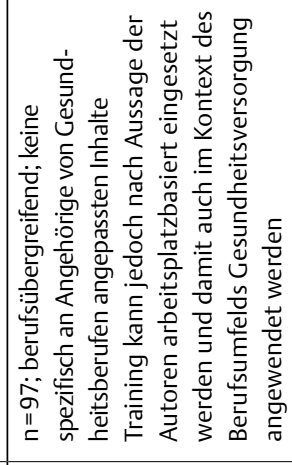 & 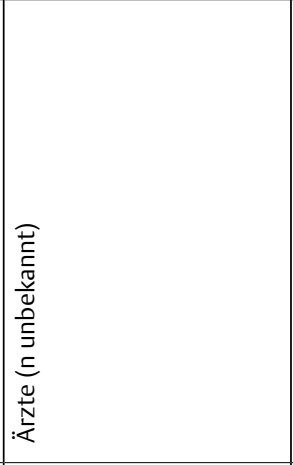 & 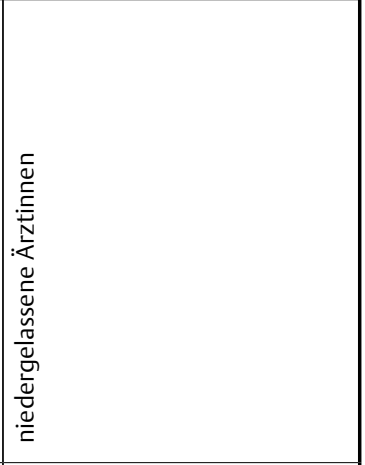 \\
\hline & 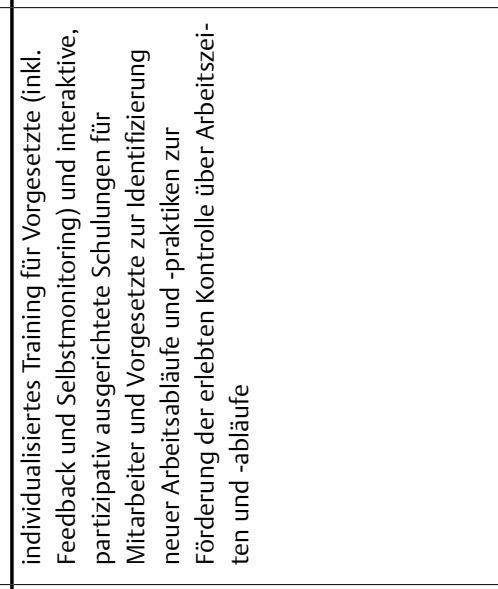 & 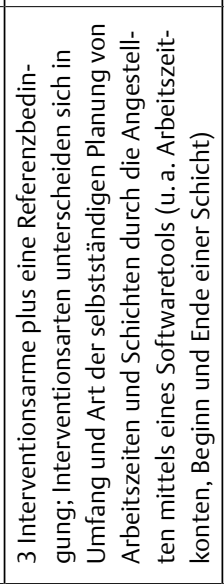 & 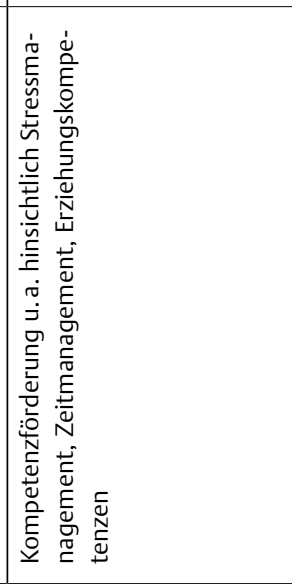 & 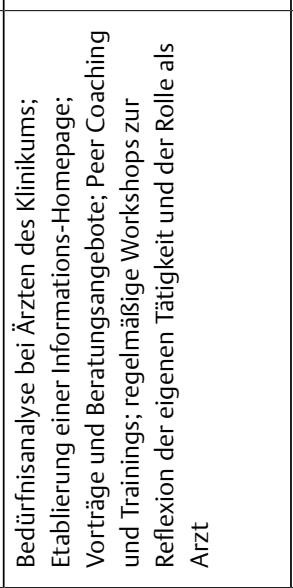 & 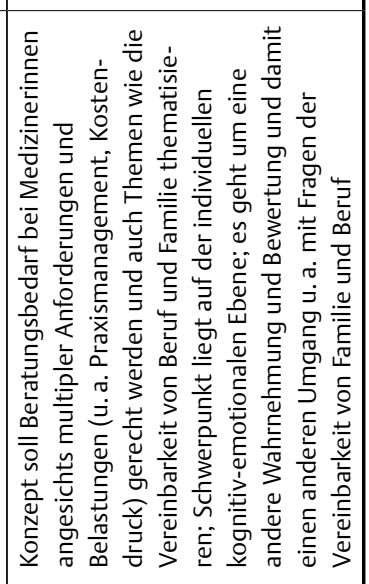 \\
\hline & 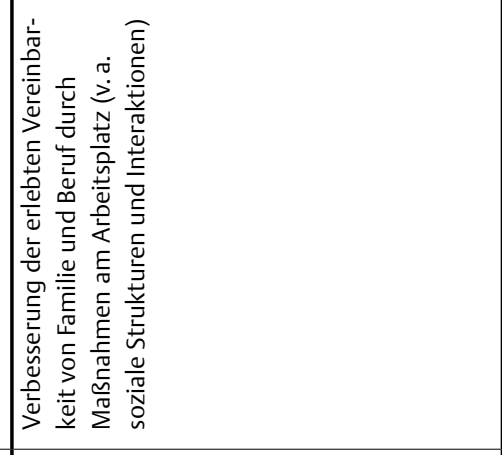 & 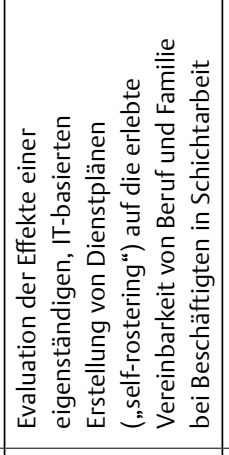 & 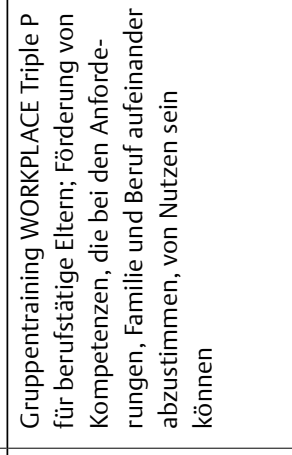 & 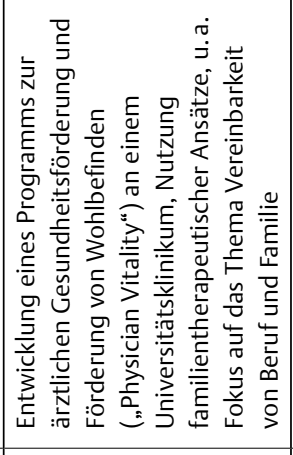 & 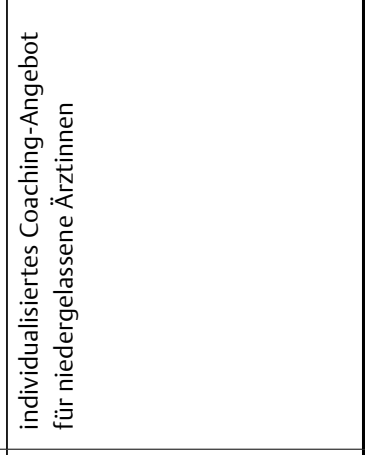 \\
\hline & 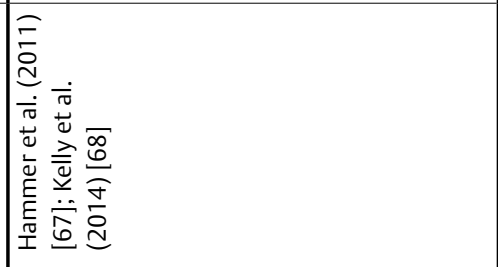 & 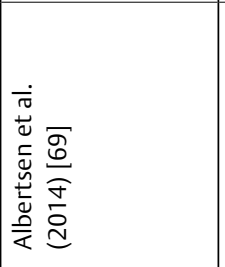 & 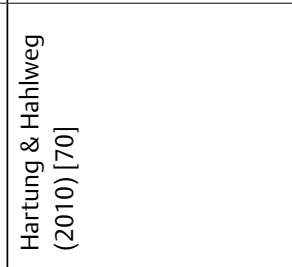 & 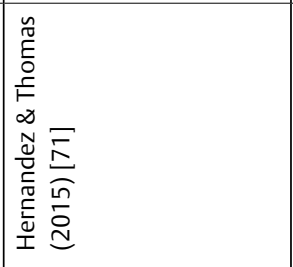 & 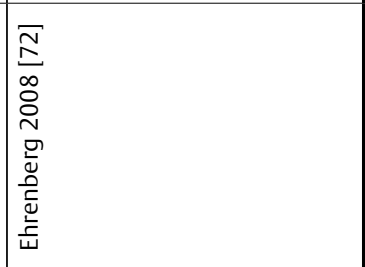 \\
\hline
\end{tabular}


Mehrere Projekte zur Förderung familienfreundlicherer Strukturen in der Medizin, die vorrangig auf der Ebene der Organisation bzw. Organisationsentwicklung anzusiedeln sind, wurden im Report Versorgungsforschung zu Arbeitsbedingungen und Befinden von Ärztinnen und Ärzten beschrieben [73]. Skizziert werden Analysen der Arbeitssituation und Veränderungen der Arbeitsbedingungen bzw. Arbeits- und Aufgabengestaltung von Ärzten in Krankenhäusern $[74,75]$ und der Einsatz von Assessments zur Dokumentation der Mitarbeitergesundheit (u. a. strukturelle Rahmenbedingungen, Prozesse, Arbeitsorganisation) als Ausgangspunkt für Maßnahmen der Organisationsentwicklung oder betrieblichen Gesundheitsförderung [76]. Zudem werden Unterstützungsangebote hinsichtlich der Vereinbarkeit von Familie und Beruf durch den Arbeitgeber beschrieben (z. B. Fort- und Weiterbildungsmöglichkeiten während der Elternzeit, kurzfristige Vertretungsmöglichkeiten, frühere Rückkehr aus der Elternzeit) [77].

\section{Diskussion und Fazit}

Mit der hier vorgestellten Übersichtsarbeit konnte erstmals ein umfassender Überblick des Forschungsstands zur Thematik „Vereinbarkeit von Familie und Gesundheitsberuf" gegeben werden. Es wurde anhand der Sichtung der Literatur erkennbar, dass hierzu wesentliche Forschungsdefizite bzw. -bedarfe bestehen. Ferner wurde deutlich, dass die Umsetzung von künftigen Forschungsaktivitäten in Form methodisch hochwertiger Studien erfolgen sollte.

Als Fazit kann zunächst festgehalten werden, dass es zu diesem Bereich sowohl national als auch international nur wenig systematische Forschung gibt. Die verfügbare Literatur fokussiert die Berufsgruppen der Ärzte (insbesondere im stationären Bereich) und beruflich Pflegenden. Die methodische Qualität der Studien ist meist gering, es handelt sich überwiegend um Querschnittstudien oder Ein-Gruppen-Prä-Post-Designs. Entsprechend finden sich keine Metaanalysen, die den bisherigen Forschungsstand zusammenfassen und z. B. einen quantitativen Vergleich der Wirksamkeit verschiedener Interventionsansätze ermöglichen.

Auch konnten nur wenige Interventionsstudien gefunden werden, die eine Verbesserung der Vereinbarkeit von Familie und Beruf als Zielgröße anstreben. Die identifizierten Studien sind nur teilweise spezifisch auf Gesundheitsberufe ausgerichtet. Zudem zeigte sich, dass in der Literatur zur Verbesserung der Gesundheitsversorgung in ländlichen Gebieten bislang keine systematische Verbindung mit Aspekten der Vereinbarkeit von Familie und Gesundheitsberuf stattgefunden hat.

Die oben skizzierten Befragungen von Ärzten und beruflich Pflegenden in Deutschland zeigen übereinstimmend die hohe Bedeutung, die der Vereinbarkeit von Familie und Gesundheitsberuf beigemessen wird. Das bloße Vorhandensein von Maßnahmen, die der Vereinbarkeit von Familie und Beruf dienen sollen, scheint dabei jedoch keine hinreichende Bedingung für eine gelingende Entwicklung und Inanspruchnahme solcher Maßnahmen zu sein. So zeigte eine Studie aus Großbritannien, dass die formale (über Statistiken usw. dokumentierte) Verfügbarkeit von Maßnahmen zur Vereinbarkeit von Beruf und Familie in Unternehmen oder Organisationen nur gering damit korreliert war, wie Mitarbeiter tatsächlich die Nutzungsmöglichkeiten solcher Maßnahmen einschätzen [78].
Als eine wesentliche Limitation der vorliegenden Arbeit ist zu nennen, dass die Aufbereitung der verfügbaren Evidenz nicht in Form eines systematischen Reviews erfolgt ist. Die entsprechenden Empfehlungen (z. B. PRISMA-Statement [79]) konnten angesichts des heterogenen und konzeptuell breit aufgefächerten Themenfelds nicht angemessen umgesetzt werden. Die Aussagekraft der Übersicht ist dementsprechend eingeschränkt. Vielmehr orientierte sich das Vorgehen an den Empfehlungen zur Evidenzaufbereitung und -darstellung im Sinne eines Scoping Review [9-11]. Dieses Vorgehen erschien zur Zusammenfassung des Wissensstands zu diesem Themenfeld sinnvoll und zielführend.

Die Übersicht kann als Ausgangspunkt verstanden werden, um den Forschungsstand noch differenzierter darzustellen und Ansatzpunkte zu identifizieren für Initiativen in der Praxis des Versorgungssystems. Eine Reihe möglicher Handlungsfelder ist denkbar, in denen Maßnahmen zur Förderung der Vereinbarkeit von Familie und Beruf im Gesundheitswesen realisiert werden können. So wurden bspw. begleitend zur Erstellung der Expertise, auf der diese Arbeit basiert [8], Ansatzpunkte in einer Arbeitsgruppe der LAGeV diskutiert und konkretisiert. Hierzu gehören die Absicherung bei Selbstständigkeit, Angehörigenbetreuung und -pflege (Double/ Triple Duty Caregiving), Arbeitsgestaltung und Arbeitszeiten, eine Angebotsinfrastruktur für das Familienleben und Kinderbetreuung und der Wiedereinstieg in den Beruf nach Elternzeit.

Angesichts der oben dargestellten Informationsdefizite ist es jedoch zunächst notwendig, eine „Voraussetzungsprüfung“ in Form von Bestands- oder Bedarfsanalysen durchzuführen, bevor Modellvorhaben in diesen oder anderen Handlungsbereichen entwickelt, implementiert und evaluiert werden. Wesentlich erscheint auch, an relevanten politischen und gesetzlichen Rahmenbedingungen anzusetzen, da diese wesentliche Voraussetzungen oder förderliche (bzw. hemmende) Strukturen für die Umsetzung von Veränderungen oder Initiativen darstellen (vgl. z. B. [80] zur Umsetzung von Delegationsmodellen).

Wie erwähnt, ist die Aussagekraft vieler vorhandener Studien begrenzt, da die methodische Qualität in der Regel gering ist. Daher sollte in Interventionen bzw. Modellprojekten eine begleitende Evaluation sichergestellt sein, idealerweise auch eine Effektprüfung. Hierzu bedarf es angesichts der Komplexität der Thematik und der unterschiedlichen Ebenen, an denen Interventionen ansetzen können, einer genauen Beschreibung und Auswahl geeigneter Outcomes, die durch eine Intervention auch veränderbar sind bzw. deren Veränderung sich dokumentieren lässt. Hierzu zählen z. B. das subjektive Vereinbarkeits- bzw. Konflikterleben oder auch psychosoziale Ressourcen. Die Bedeutung arbeitsbezogener und individueller Ressourcen für die Reduktion von Konflikterleben wurde in einer deutschen Studie mit Klinikärzten belegt [81]. Eine innovative Perspektive, die in künftigen Studien aufgegriffen werden könnte, wäre die Untersuchung der Frage, inwieweit dem Versorgungsmangel in ländlichen Regionen über eine Verbesserung der Vereinbarkeit von Gesundheitsberuf und Familie entgegengewirkt werden kann.

Interessenkonflikt

Die Autoren geben an, dass kein Interessenkonflikt besteht. 
[1] Amstad FT, Meier LL, Fasel U et al. A meta-analysis of work-family conflict and various outcomes with a special emphasis on cross-domain versus matching-domain relations. J Occup Health Psychol 2011; 16: 151-169; doi:10.1037/a0022170

[2] Byron K. A meta-analytic review of work-family conflict and its antecedents. J Vocat Behav 2005; 67: 169-198; doi:dx.doi. org/10.1016/j.jvb.2004.08.009

[3] Ford MT, Heinen BA, Langkamer KL. Work and family satisfaction and conflict: A meta-analysis of cross-domain relations. J Appl Psychol 01: 2007; 92: 57-80; doi:https://dx.doi.org/10.1037/0021-9010.92.1.57

[4] Michel JS, Mitchelson JK, Kotrba LM et al. A comparative test of work-family conflict models and critical examination of work-family linkages. J Vocat Behav 2009; 74: 199-218; doi:10.1016/j. jvb.2008.12.005

[5] Cinamon RG, Weisel A, Tzuk K. Work-family conflict within the family: Crossover effects, perceived parent-child interaction quality, parental self-efficacy, and life role attributions. J Career Dev 2007; 34: 79-100; doi:10.1177/0894845307304066

[6] Greenhaus JH, Beutell NJ. Sources of conflict between work and family roles. Acad Manage Rev 1985; 10: 76-88

[7] Hammer LB, Cullen JC, Neal MB et al. The longitudinal effects of work-family conflict and positive spillover on depressive symptoms among dual-earner couples. J Occup Health Psychol 2005; 10: 138-154; doi:https://dx.doi.org/10.1037/1076-8998.10.2.138

[8] Lukasczik M, Ahnert ], Ströbl V et al. Vereinbarkeit von Familie und Gesundheitsberuf unter besonderer Berücksichtigung des ländlichen Raums. Band 1 der Schriftenreihe Versorgungsforschung. Erlangen: Bayerisches Landesamt für Gesundheit und Lebensmittelsicherheit (LGL) (Hrsg.); 2016

[9] Colquhoun HL, Levac D, O'Brien KK et al. Scoping reviews: Time for clarity in definition, methods, and reporting. J Clin Epidemiol 2014; 67: 1291-1294; doi:dx.doi.org/10.1016/j.jclinepi.2014.03.013

[10] Arksey H, O'Malley L. Scoping studies: Towards a methodological framework. Int J Soc Res Methodol 2005; 8: 19-32; doi:10.1080/1364557032000119616

[11] Schmucker C, Motschall E, Antes G et al. Methoden des Evidence Mappings: Eine systematische Übersichtsarbeit. Bundesgesundheitsbl 2013; 56: 1390-1397; doi:10.1007/s00103-013-1818-y

[12] Adám S, Györffy Z, Susánszky E. Physician burnout in Hungary: A potential role for work-family conflict. J Health Psychol 2008; 13: 847-856; doi:10.1177/1359105308095055

[13] Ahmad A. Work-family conflict among junior physicians: Its mediating role in the relationship between role overload and emotional exhaustion. J Soc Sci 2010; 6: 265-271; doi:10.3844/jssp.2010.265.271

[14] Estryn-Behar M, Fry C, Guetarni K et al. Work week duration, work-family balance and difficulties encountered by female and male physicians: Results from the French SESMAT study. Work 2011; 40: (Suppl 1): S83-S100; doi:10.3233/WOR-2011-1270

[15] Geurts S, Rutte C, Peeters M. Antecedents and consequences of work-home interference among medical residents. Soc Sci Med 1999; 48: $1135-1148$

[16] Thanacoody PR, Bartram T, Casimir G. The effects of burnout and supervisory social support on the relationship between work-family conflict and intention to leave: A study of Australian cancer workers. J Health Organ Manag 2009; 23: 53-69; doi:10.1108/14777260910942551

[17] Berkman LF, Liu SY, Hammer L et al. Work-family conflict, cardiometabolic risk, and sleep duration in nursing employees. J Occup Health Psychol 2015 doi:10.1037/a0039143

[18] Cortese CG, Colombo L, Ghislieri C. Determinants of nurses' job satisfaction: The role of work-family conflict, job demand, emotional charge and social support. J Nurs Manag 2010; 18: 35-43; doi:10.1111/j.1365-2834.2009.01064.x
[19] Kim SS, Okechukwu CA, Buxton OM et al. Association between work-family conflict and musculoskeletal pain among hospital patient care workers. Am J Ind Med 2013; 56: 488-495; doi:10.1002/ajim.22120

[20] Leineweber C, Westerlund H, Chungkham HS et al. Nurses' practice environment and work-family conflict in relation to burn out: A multilevel modelling approach. PLoS ONE 2014; 9: e96991; doi:10.1371/journal.pone.0096991

[21] Simon M, Kümmerling A, Hasselhorn H. Work-home conflict in the European nursing profession. Int J Occup Environ Health 2004; 10: 384-391; doi:10.1179/oeh.2004.10.4.384

[22] Wilson MG, Dejoy DM, Vandenberg RJ et al. Work characteristics and employee health and well-being: Test of a model of healthy work organization. J Occup Organ Psychol 2004; 77: 565-588; doi:10.1348/0963179042596522

[23] Stordeur S, D’Hoore W. NEXT Study Group. Organizational configuration of hospitals succeeding in attracting and retaining nurses. J Adv Nurs 2007; 57: 45-58; doi:10.1111/j.1365-2648.2006.04095.x

[24] DePasquale N, Davis KD, Zarit SH et al. Combining formal and informal caregiving roles: The psychosocial implications of double- and triple-duty care. J Gerontol B Psychol Sci Soc Sci 2016; 1: 201-211; doi:10.1093/geronb/gbu139

[25] Ward-Griffin C, Brown JB, St-Amant O et al. Nurses negotiating professional-familial care boundaries: Striving for balance within double duty caregiving. J Fam Nurs 2015; 21: 57-85; doi:10.1177/1074840714562645

[26] DePasquale N, Bangerter LR, Williams J et al. Certified nursing assistants balancing family caregiving roles: Health care utilization among double- and triple-duty caregivers. Gerontologist 2015; doi:10.1093/geront/gnv081

[27] Boumans NPG, Dorant E. Double-duty caregivers: Healthcare professionals juggling employment and informal caregiving. A survey on personal health and work experiences. J Adv Nurs 2014; 70: 1604-1615; doi:10.1111/jan.12320

[28] Fuss I, Nubling M, Hasselhorn HM et al. Working conditions and work-family conflict in German hospital physicians: Psychosocial and organisational predictors and consequences. BMC Public Health 2008; 8: 353; doi:10.1186/1471-2458-8-353

[29] Rammoser I, Lüthy A, Ehret T. Pflege: Unzufriedenheit wächst dramatisch. Health \& Care Management 2012

[30] Buxel $\mathrm{H}$. Jobwahlverhalten, Motivation und Arbeitsplatzzufriedenheit von Pflegepersonal und Auszubildenden in Pflegeberufen: Ergebnisse dreier empirischer Untersuchungen und Implikationen für das Personalmanagement und -marketing von Krankenhäusern und Altenpflegeeinrichtungen. 2011. https://fh-muenster.de/oecotrophologie-facility-management/downloads/holger-buxel/2011_Studie_ Zufriedenheit_Pflegepersonal.pdf; Letzter Zugriff am 28.10.2016

[31] Galatsch M, Iskenius M, Müller BH et al. Vergleich der Gesundheit und Identifizierung von Prädiktoren der Gesundheit in verschiedenen Altersgruppen Pflegender in Deutschland. Pflege 2012; 25: 23-32; doi:10.1024/1012-5302/a000167

[32] Engelmann C. Vater werden ist nicht schwer,... Vater sein dagegen sehr: Elternzeit: Wirklichkeit als Inspiration für Forschung. Deutsches Ärzteblatt 2015; 112

[33] Gensch K. Berufsentscheidung junger Ärztinnen und Ärzte: Auswirkungen auf das ärztliche Versorgungsangebot. In: Schwartz $F$, Angerer $P$ editors. Arbeitsbedingungen und Befinden von Ärztinnen und Ärzten: Befunde und Interventionen. Köln: Deutscher Ärzte-Verlag; 2010: 127-136

[34] Bühren A. Ärztinnen und Ärzte im Gleichgewicht - Beruf, Familie, Freizeit und Gesundheit. In: Schwartz F, Angerer P editors. Arbeitsbedingungen und Befinden von Ärztinnen und Ärzten: Befunde und Interventionen. Köln: Deutscher Ärzte-Verlag; 2010: 17-29 
[35] Hartmannbund. Assistenzarzt (m/w) 2014. 2014; http://www. hartmannbund.de/fileadmin/user_upload/Downloads/Umfragen/ 2015_Assistenzarztumfrage2014.pdf; Letzter Zugriff am 28.10.2016

[36] Institut für freie Berufe Nürnberg (IFB). Zahnärztinnen in Bayern Vereinbarkeit von Familie und Beruf. http://www.gbv.de/dms/ zbw/647684055.pdf; Letzter Zugriff am 28.10.2016

[37] Bestmann T, Küchler T, Henne-Bruns D. Zufriedenheit unter deutschen Ärztinnen und Ärzten- Ergebnisse einer empirischen Umfrage. In: Schwartz F, Angerer P editors. Arbeitsbedingungen und Befinden von Ärztinnen und Ärzten: Befunde und Interventionen. Köln: Deutscher Ärzte-Verlag; 2010: 209-218

[38] Heyer T. Ergebnisse der Online-Befragung zur Kinderbetreuung. Rheinisches Zahnärzteblatt 2014; 9: 508-510

[39] Flaig T. Vereinbarkeit von Familie und Beruf für Ärztinnen und Ärzte der Medizinischen Fakultät. Dissertation, Universität Ulm. 2014; http://vts.uni-ulm.de/docs/2014/9308/vts_9308_13995.pdf; Letzter Zugriff am 28.10.2016

[40] Bühren A, Buchalik M. Praxis und Familie - selbstverständlich oder Problem? Praxisrelevante Ergebnisse einer Umfrage. Hausarzt 2008; 45: $19-20$

[41] Bühren A, Tschörtner A. Ich bin Ärztin. 2011; https://www.thieme.de/ statics/dokumente/thieme/final/de/dokumente/zw_xx/lch_bin_ Aerztin.pdf; Letzter Zugriff am 28.10.2016

[42] Fischer ]. Der Ärztemonitor 2014: Zwischen Lust und Frust an Rhein und Ruhr 2014; http://www.aekno.de/page.asp?pageld=11479\&noredir=True; Letzter Zugriff am 28.10.2016

[43] Deutsche Gesellschaft für Kardiologie. Vereinbarkeit von Beruf und Familie in der Kardiologie. 2014; http://www.kardiologie.org/ vereinbarkeit-von-beruf-und-familie-in-der-kardiologie/37588; Letzter Zugriff am 28.10.2016

[44] Hancke K, Toth B, Igl W et al. Career and family - Are they compatible? Results of a survey of male and female gynaecologists in Germany. Geburtshilfe Frauenheilkd 2012; 72: 403-407; doi:10.1055/ s-0031-1298393

[45] Hartmannbund. Es bleibt noch viel zu tun! http://www.hartmannbund. de/berufspolitik/informationen/umfragen/assistenzarzt-mw-2014/; Letzter Zugriff am 28.10.2016

[46] Flintrop J. Unzufrieden in der Klinik - Respekt vor der Niederlassung. Deutsches Ärzteblatt 2015; 112: 216-217

[47] Oberlander W. Die berufliche Situation junger Ärztinnen und Ärzte. In: Schwartz F, Angerer P editors. Arbeitsbedingungen und Befinden von Ärztinnen und Ärzten: Befunde und Interventionen. Köln: Deutscher Ärzte-Verlag; 2010: 117-126

[48] Barth N, Linde K, Schneider A. Niederlassungsmotive - Die Bereitschaft zur Niederlassung in eigener Praxis von Ärztinnen und Ärzten in Weiterbildung zum Facharzt für Allgemeinmedizin. Gesundheitswesen 2015, doi:10.1055/s-0035-1547299

[49] Bayerische Landesärztekammer. Tätigkeitsbericht der Bayerischen Landesärztekammer 2014/15 dem 74. Bayerischen Ärztetag vorgelegt. 2015; http://www.blaek.de/werwaswo/bay_aerztetag/berichte/TB \%20 2014_15.pdf; Letzter Zugriff am 28.10.2016

[50] Institut für freie Berufe Nürnberg, Bayerische Landeszahnärztekammer. Gender dentistry - Ergebnisse der Umfrage der Bayerischen Landeszahnärztekammer unter Zahnärztinnen 2010. http://www. bzb-online.de/jan11/22_23.pdf; Letzter Zugriff am 28.10.2016

[51] Deutscher Ärztinnenbund e.V. Befragung des Deutschen Ärztinnenbunds zur Kinderbetreuung an deutschen Kliniken und Krankenhäusern - Abschlussbericht 2. 2006. http://www.aerztinnenbund.de/ Befragung-des-Deutschen-rztinnenbunds-zur.649.0.2.html; Letzter Zugriff am 28.10.2016
[52] Blum K, Löffert S. Ärztemangel im Krankenhaus - Ausmaß, Ursachen, Gegenmaßnahmen. Forschungsgutachten im Auftrag der Deutschen Krankenhausgesellschaft. 2010; http://www.dkgev.de/media/ file/8324.2010_10_11_Aerztemangel_Endbericht_1.pdf; Letzter Zugriff am 28.10.2016

[53] Blum K, Offermanns M, Perner P. Krankenhaus Barometer 2008; https://www.dki.de/sites/default/files/publikationen/bericht_kh_ barometer_2008.pdf; Letzter Zugriff am 28.10.2016

[54] DIHK Berlin, Christian-Albrechts-Universität zu Kiel \& dem Bundesverband privater Anbieter sozialer Dienste. Ergebnispräsentation „Vereinbarkeit von Beruf und Familie in Altenpflegeeinrichtungen“. http://www.competentia.nrw.de/interessantes/publikationen/140909_Vereinbarkeit-altenpflege.pdf; Letzter Zugriff am 28.10.2016

[55] Rygh EM, Hjortdahl P. Continuous and integrated health care services in rural areas. A literature study. Rural Remote Health 2007; 7: 766

[56] Kane KY, Quinn KJ, Stevermer J] et al. Summer in the country: Changes in medical students' perceptions following an innovative rural community experience. Acad Med 2013; 88: 1157-1163; doi:10.1097| ACM.0b013e318299fb5d

[57] Wilson M, Cleland J. Evidence for the acceptability and academic success of an innovative remote and rural extended placement. Rural Remote Health 2008; 8: 960

[58] McAuliffe T, Barnett F. Perceptions towards rural and remote practice: a study of final year occupational therapy students studying in a regional university in Australia. Aust Occup Ther J 2010; 57: 293-300 doi:10.1111/j.1440-1630.2009.00838.x

[59] Roberts A, Heaney D, Haddow G et al. Implementation of a national, nurse-led telephone health service in Scotland: Assessing the consequences for remote and rural localities. Rural Remote Health 2009; 9: 1079

[60] Langer A, Ewert T, Hollederer A et al. Literaturüberblick über niederlassungsfördernde und -hemmende Faktoren bei Ärzten in Deutschland und daraus abgeleitete Handlungsoptionen für Kommunen. Gesundheitsökonomie Qualitätsmanagement 2015; 20: 11-18; doi:dx.doi.org/10.1055/s-0033-1356303

[61] van den Berg N, Fiss T, Meinke C et al. GP-support by means of AGnES-practice assistants and the use of telecare devices in a sparsely populated region in Northern Germany - proof of concept. BMC Fam Pract 2009; 10: 44; doi:10.1186/1471-2296-10-44

[62] van den Berg N, Heymann R, Meinke $C$ et al. Effect of the delegation of GP-home visits on the development of the number of patients in an ambulatory healthcare centre in Germany. BMC Health Serv Res 2012; 12: 355; doi:10.1186/1472-6963-12-355

[63] Joyce K, Pabayo R, Critchley JA et al. Flexible working conditions and their effects on employee health and wellbeing. Cochrane Database Syst Rev 2010, CD008009; doi:10.1002/14651858.CD008009.pub2

[64] Allen TD, Johnson RC, Kiburz KM et al. Work-family conflict and flexible work arrangements: Deconstructing flexibility. Pers Psychol 2013; 66: 345-376; doi:10.1111/peps.12012

[65] Higgins C, Duxbury L, Julien M. The relationship between work arrangements and work-family conflict. Work 2014; 48: 69-81; doi:10.3233/WOR-141859

[66] Kürschner I, Strobel M, Tumasjan A et al. Arbeits- und Lebensgestaltung der Zukunft: Individuelle und institutionelle Einflussfaktoren Chancengleichheit auf dem Arbeitsmarkt und Vereinbarkeit von Familie und Beruf in Bayern. München: Hanns Seidel Stiftung; 2012

[67] Hammer LB, Kossek EE, Anger WK et al. Clarifying work-family intervention processes: The roles of work-family conflict and family-supportive supervisor behaviors. J Appl Psychol 2011; 96: 134-150; doi:10.1037/a0020927 
[68] Kelly EL, Moen P, Oakes JM et al. Changing work and work-family conflict: evidence from the Work, Family, and Health Network*. Am Sociol Rev 2014; 79: 485-516; doi:10.1177/0003122414531435

[69] Albertsen K, Garde AH, Nabe-Nielsen K et al. Work-life balance among shift workers: Results from an intervention study about self-rostering. Int Arch Occup Environ Health 2014; 87: 265-274; doi:10.1007| s00420-013-0857-x

[70] Hartung D, Hahlweg K. Strengthening parent well-being at the work-family interface: A German trial on workplace Triple P. J Community Appl Soc Psychol 2010; 20: 404-418; doi:10.1002/casp.1046

[71] Hernandez BC, Thomas TL. The development of a physician vitality program: A brief report. J Marital Fam Ther 2015; 41: 443-450; doi:10.1111/jmft.12085

[72] Ehrenberg B. Coaching für niedergelassene Ärztinnen Ein kognitivverhaltenstherapeutischer Ansatz. OSC Organisationsberatung Supervision Coaching 2008; 15: 374-384; doi:10.1007/s11613-0080095-3

[73] Schwartz FW, Angerer P. editors. Arbeitsbedingungen von Ärztinnen und Ärzten - Befunde und Interventionen: Report Versorgungsforschung, Band 2. Köln: Deutscher Ärzte-Verlag; 2010

[74] Ulich E. Arbeit und Befinden von Ärzten und Prinzipien gesundheitsorientierter Arbeitsgestaltung im Krankenhaus. In: Schwartz FW, Angerer P.editors. Arbeitsbedingungen von Ärztinnen und Ärzten - Befunde und Interventionen: Report Versorgungsforschung, Band 2. Köln: Deutscher Ärzte-Verlag; 2010: 345-358

[75] Weigl M, Glaser J, Hornung S et al. Gestaltung ärztlicher Arbeit im Krankenhaus: Intervention und Evaluation. In: Schwartz FW, Angerer P.editors. Arbeitsbedingungen von Ärztinnen und Ärzten - Befunde und Interventionen: Report Versorgungsforschung, Band 2. Köln: Deutscher Ärzte-Verlag; 2010: 359-372
[76] Karl-Trummer U. Arbeitsbedingungen und Arbeitsbelastungen von Mitarbeiterinnen und Mitarbeitern im Krankenhaus - Ärztinnen und Ärzte im Vergleich mit anderen Berufsgruppen. In: Schwartz FW, Angerer P. editors. Arbeitsbedingungen von Ärztinnen und Ärzten Befunde und Interventionen: Report Versorgungsforschung, Band 2. Köln: Deutscher Ärzte-Verlag; 2010: 373-384

[77] Zendler M, Krug S. Gestaltung ärztlicher Arbeitsbedingungen: Arbeitsgestaltung aus Sicht der Kaufmännischen Leitung. In: Schwartz FW, Angerer P.editors. Arbeitsbedingungen von Ärztinnen und Ärzten - Befunde und Interventionen: Report Versorgungsforschung, Band 2. Köln: Deutscher Ärzte-Verlag; 2010: 403-410

[78] Budd JW, Mumford KA. Family-friendly work practices in Britain: Availability and perceived accessibility. Hum Resour Manage 2006; 45: 23-42; doi:10.1002/hrm.20091

[79] Moher D, Liberati A, Tetzlaff J et al.The PRISMA Group. Preferred reporting items for systematic reviews and meta-analyses: The PRISMA statement. PLoS Med 2009; 6: e1000097; doi:10.1371/ journal.pmed.1000097

[80] Ruppel T, van den Berg N, Hoffmann W. Juristische Analyse der Umsetzungsregelungen der Delegation vertragsärztlicher Hausbesuche an nicht-ärztliche Gesundheitsberufe - ist die Umsetzung in Einklang mit dem Willen des Gesetzgebers? Gesundheitswesen 2016; 78: 622-627; doi:10.1055/s-0042-108646

[81] Mache S, Bernburg M, Vitzthum K et al. Managing work-family conflict in the medical profession: Working conditions and individual resources as related factors. BMJ Open 2015; 5: e006871; doi:10.1136/ bmjopen-2014-006871 